\title{
Feasibility study of endoscopic biliary drainage under direct peroral cholangioscopy by using an ultra-slim upper endoscope (with videos)
}

\section{다(1) $(9)$}

\author{
Authors \\ Deok $\mathrm{Cho}^{3}$, Sang-Heum Park ${ }^{2}$ \\ Institutions \\ 1 Digestive Disease Center and Research Institute, \\ Department of Internal Medicine, SoonChunHyang \\ University School of Medicine, Bucheon, Korea \\ 2 Digestive Disease Center and Research Institute, \\ Department of Internal Medicine, SoonChunHyang \\ University School of Medicine, Cheonan, Korea \\ 3 Digestive Disease Center and Research Institute, \\ Department of Internal Medicine, SoonChunHyang \\ University School of Medicine, Seoul, Korea
}

Jae Keun Park' ${ }^{1}$ Jong Ho Moon¹, Yun Nah Lee ${ }^{1}$, Seok jung Jo ${ }^{1}$, Moon Han Choi ${ }^{1}$, Tae Hoon Lee², Sang-Woo Cha ${ }^{3}$, Young

submitted 25.2.2020

accepted after revision 30.12 .2020

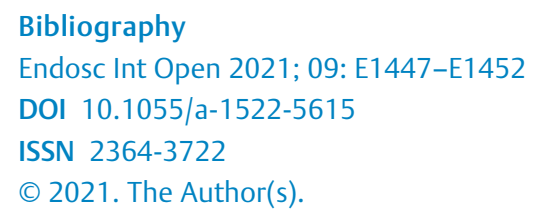

This is an open access article published by Thieme under the terms of the Creative Commons Attribution-NonDerivative-NonCommercial License, permitting copying and reproduction so long as the original work is given appropriate credit. Contents may not be used for commercial purposes, or adapted, remixed, transformed or built upon. (https://creativecommons.org/licenses/by-nc-nd/4.0/)

Georg Thieme Verlag KG, Rüdigerstraße 14,

70469 Stuttgart, Germany

Corresponding author

Jong Ho Moon, MD, PhD, Department of Internal Medicine, SoonChunHyang University School of Medicine, Digestive Disease Center and Research Institute, SoonChunHyang
University Bucheon Hospital, 170 jomaru-ro, Wonmi-gu,

Bucheon, 14584, Korea

Fax: +82-32-621-5018

jhmoon@schmc.ac.kr

\section{ABSTRACT}

The therapeutic utility of peroral cholangioscopy (POC) is limited. Direct POC using an ultra-slim upper endoscope expands the therapeutic indications because of its larger working channel, of up to $2.2 \mathrm{~mm}$. We evaluated the feasibility of selective biliary drainage using a plastic stent under direct POC. From April 2015 to March 2019, biliary drainage under endoscopic visualization was performed in the same endoscopic session as direct POC without exchanging the duodenoscope. After guidewire insertion through the stricture or stone, a $5 \mathrm{Fr}$ plastic stent and/or nasobiliary drainage catheter was used for biliary drainage. Selective biliary drainage under direct POC was performed in 32 patients, including 17 with difficult bile duct stones. Biliary drainage was performed with a plastic stent in 29 patients, nasobiliary drainage in one, and combined drainage in two patients. The technical success rate for biliary drainage placement under direct POC was $100 \%$ (32/32). No significant procedure-related complications occurred. In conclusion, biliary drainage with a plastic stent or catheter under direct POC using an ultra-slim upper endoscope is feasible and may be useful for lesions obstructing the bile duct.

\section{Introduction}

Peroral cholangioscopy (POC) enables not only endoscopic direct visualization of the biliary system, but also interventions for diseases of the bile duct [1]. POC can be achieved using a conventional mother baby scope system, or the single operator cholangioscopy system with the SpyGlass Direct Visualization system or direct POC using an ultra-slim upper endoscope [1]. Selective guidewire access through complex strictures or stones, and subsequent stent insertion under direct visualization, may increase the therapeutic utility of POC. However, mother-baby endoscope system and the SpyGlass direct visualization system have working channels of only $1.2 \mathrm{~mm}$. Although selective guidewire advancement is possible, direct biliary stent placement is impossible using endoscopes with such small working channels. In contrast, direct POC using an ultra-slim upper endoscope with an up to 2.2-mm working channel ex- 
pands the therapeutic indications for direct POC. We evaluated the feasibility of selective biliary drainage under direct POC.

\section{Patients and methods \\ Patients and study design}

This retrospective analysis of a prospectively maintained database study was performed in a single tertiary referral center from April 2015 to March 2019. The inclusion criteria were: (1) clinical symptoms of bile duct obstruction with jaundice; (2) diameter of the distal common bile duct (CBD) of $\geq 8 \mathrm{~mm}$; (3) endoscopic sphincteroplasty and/or endoscopic papillary balloon dilation; and (4) need for direct POC. The exclusion criteria were: (1) age <18 years; (2) contradictions for endoscopic retrograde cholangiopancreatography (ERCP); (3) bleeding tendency (platelet count of $<50,000 / \mathrm{mm}^{3}$ or prothrombin time [PT] international normalised ratio [INR] of $>1.5$ ); and (4) refusal to participate. Written informed consent for the endoscopic procedure was obtained from all of the enrolled patients. The Institutional Review Board of Soonchunhyang Medical Center approved the study protocol.

\section{Instruments}

The ultra-slim upper endoscopes were the GIF-XP260N, GIFXP260NS, and GIF-XP290N instruments (Olympus Medical Systems, Co., Ltd., Tokyo, Japan), which have distal tips 5.0 to $5.5 \mathrm{~mm}$ in diameter. All of these endoscopes are forward-viewing type, featuring a 2.0- to 2.2-mm-diameter working channel and four-way deflection steering of the tip. A 5-Fr balloon catheter (MTW Endoskopie, Wesel, Germany) was used to assist the cholangioscopy [2]. For biliary drainage, 5-Fr plastic stents or nasobiliary drainage catheters were used (Cook Endoscopy, Winston-Salem, North Carolina, United States).

\section{Direct POC procedure}

The patients were placed in the prone position under sedation with midazolam and/or propofol and received prophylactic antibiotics. Endoscopic sphincterotomy and/or endoscopic papillary balloon dilatation was performed before the introduction of an ultra-slim endoscope for direct POC, which was performed using a previously described intraductal balloon-guided method [2,3]. Irrigation with sterile $0.9 \% \mathrm{NaCl}$ and frequent suction were used to improve visualization during direct POC. Minimal insufflation of CO2 (Colcosense CO-3000; Mirae Medics, Co., Seoul, South Korea) was applied as needed, and room air insufflation was not used.

\section{Biliary drainage procedure}

A guidewire with a diameter of 0.025 or 0.035 inches was inserted under direct visualization through the stricture or stone. Biliary drainage was performed over the guidewire through the working channel of the ultra-slim upper endoscope using 5-Fr double-pigtail or straight plastic stent, and/or 5-Fr nasobiliary drainage catheters ( $>$ Fig. 1, $>$ Video 1, > Video 2).
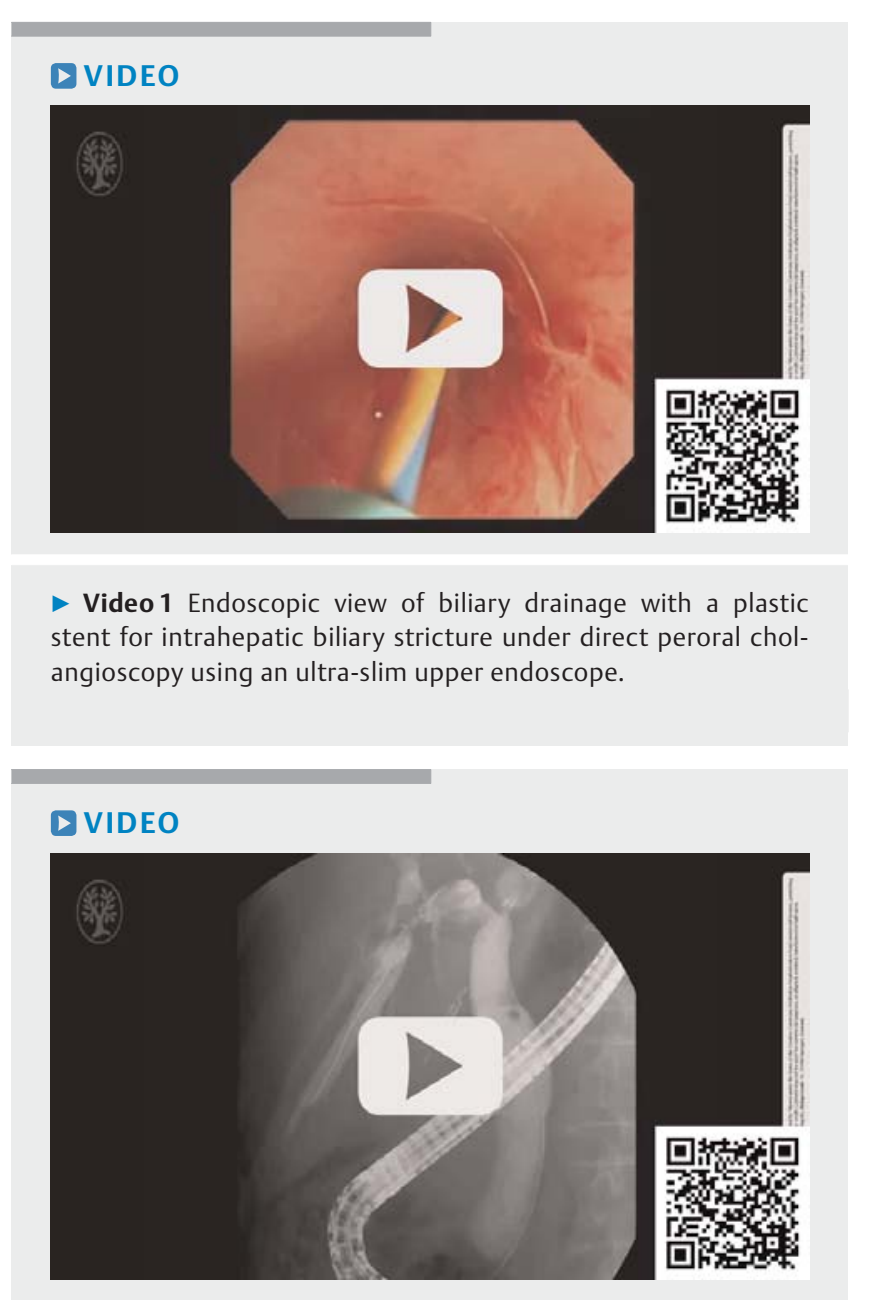

- Video 2 Fluoroscopic view of biliary drainage with a plastic stent for intrahepatic biliary stricture under direct peroral cholangioscopy using an ultra-slim upper endoscope.

\section{Definition of events and outcomes}

Technical success was defined as successful endoscopic retrograde biliary drainage (ERBD) and/or endoscopic nasobiliary drainage (ENBD) placement in the bile duct under direct POC without exchange for a duodenoscope. Functional success was defined as resolution of clinical symptoms, including jaundice, after biliary drainage without any need for repeated drainage procedures. Adverse events (AEs) after ERCP were recorded according to the guidelines of the American Society for Gastrointestinal Endoscopy [4]. The primary outcome was the technical success rate. The secondary outcomes were the functional success rate and incidence of procedure-related AEs.

\section{Statistical analysis}

Demographic and clinical characteristics are presented as means (standard deviation) for continuous variables and frequencies (percentage) for categorical variables. Statistical analysis was performed using R (version 3.3.3; R Foundation for Sta- 
- Table 1 Baseline characteristics of the patients.

\begin{tabular}{|l|c|}
\hline Characteristic & All patients (N=32) \\
\hline Mean age \pm SD, years & $71.2 \pm 11.3$ \\
\hline Sex - number (\%) & $16(50)$ \\
\hline - Male & $16(50)$ \\
\hline - Female & \\
\hline Indications for direct POC - number (\%) & $17(53.1)$ \\
\hline - Difficult CBD stones & $12(37.5)$ \\
\hline - Evaluation of indeterminate BD lesion & $3(9.4)$ \\
\hline - Tumor ablation therapy (PDT) & \\
\hline $\begin{array}{l}\text { SD, standard deviation; POC, peroral cholangioscopy; CBD, common bile } \\
\text { duct; BD, bile duct; PDT, photodynamic therapy. }\end{array}$ \\
\hline
\end{tabular}

tistical Computing, Vienna, Austria) or SPSS software (version 23.0; IBM Corp., Armonk, New York, United States).

\section{Results}

Selective biliary drainage under direct POC using an ultra-slim upper endoscope was attempted in 32 patients (16 males, 16 females; mean age, 71.2 years). The baseline characteristics of the patients are listed in $>$ Table 1 . The most common indication for direct POC was difficult bile duct stones (17 patients, $53.1 \%)$. Difficult bile duct stones were defined as larger than $15 \mathrm{~mm}$ and/or more than three stones that could not be removed despite an extended sphincterotomy and recurrent ERCP procedures may be needed [5, 6]. Biliary drainage was attempted after endoscopic intervention by direct POC guided intraductal electrohydraulic lithotripsy (EHL) ( $>$ Fig.1), tumor ablation with photodynamic therapy (PDT) to check the exact extent and perform the procedure ( $>$ Fig. 2), and indeterminate biliary stricture in case of suspected malignant bile duct stricture, but failed diagnosis through conventional ERCP-guided tissue sampling ( $\mathbf{F i g} \cdot \mathbf{3}$ ).
- Table 2 Summary of biliary drainage under direct POC.

\begin{tabular}{|c|c|}
\hline & Number $(\%)(\mathrm{N}=32)$ \\
\hline \multicolumn{2}{|c|}{ Drainage under direct $\mathrm{POC}$} \\
\hline - ERBD & $29(91)$ \\
\hline - ENBD + ENBD & $2(6)$ \\
\hline - ENBD & $1(3)$ \\
\hline Technical success & $32(100)$ \\
\hline Functional success & $32(100)$ \\
\hline Adverse events $^{1}$ & $1(3.1)$ \\
\hline \multicolumn{2}{|c|}{$\begin{array}{l}\text { POC, peroral cholangioscopy; ERBD, endoscopic retrograde biliary drainage; } \\
\text { ENBD, endoscopic nasobiliary drainage. } \\
{ }^{1} \text { Minor bleeding. }\end{array}$} \\
\hline
\end{tabular}

The technical success rate for selective biliary drainage under direct POC using an ultra-slim upper endoscope was $100 \%$. Results of selective biliary drainage under direct POC are listed in $>$ Table 2. Twenty-nine patients (91\%) underwent ERBD with $5-\mathrm{Fr}$ plastic stents. One patient (3\%) underwent only ENBD. ERBD and ENBD were performed simultaneously in two patients (6\%). The functional success rate was $100 \%$ (32/32 patients). Only one patient had minor bleeding during the procedure after fragmentation of a stone by EHL.

\section{Discussion}

A variety of clinical conditions accompanied by jaundice necessitate biliary drainage. In cases of difficult guidewire placement, POC enables direct visualization of the lesion [7]. There have been several case reports of successful guidewire placement using the SpyGlass cholangioscope [8-10]. However, although SpyGlass cholangioscopy enables direct visualization of the bile duct, its therapeutic utility is limited by the small working channel $(1.2 \mathrm{~mm})$. Thus, the SpyGlass cholangioscope must be withdrawn before inserting the stent for drainage [11].
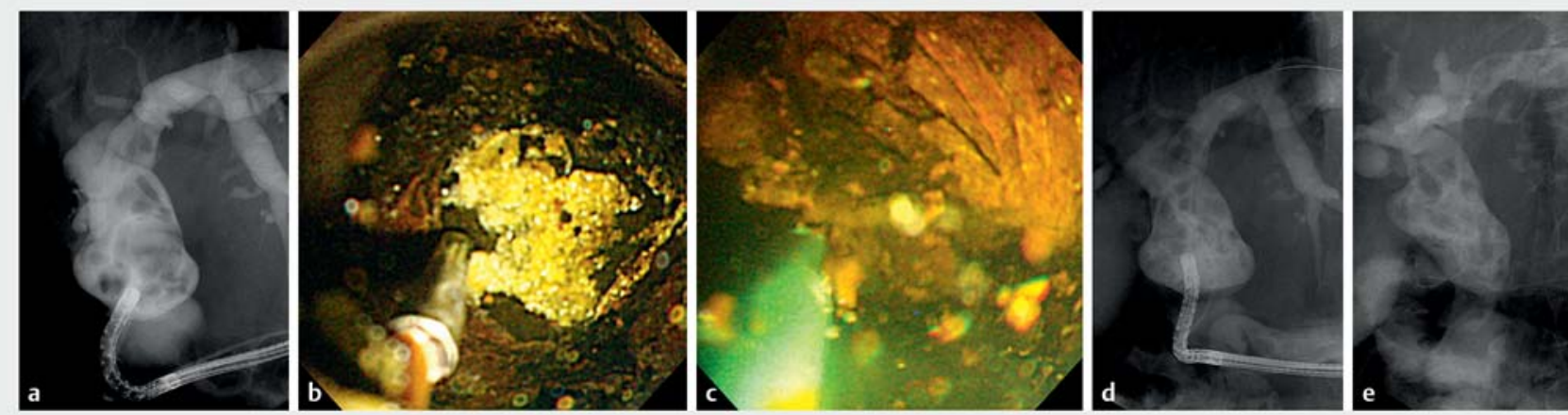

- Fig. 1 a Cholangiogram of difficult bile duct stones. b Direct peroral cholangioscopy-guided intraductal electrohydraulic lithotripsy. c A 5-Fr plastic stent was inserted under direct peroral cholangioscopy (POC). d and e Fluoroscopic findings showing endoscopic retrograde biliary drainage (ERBD) under direct POC. 

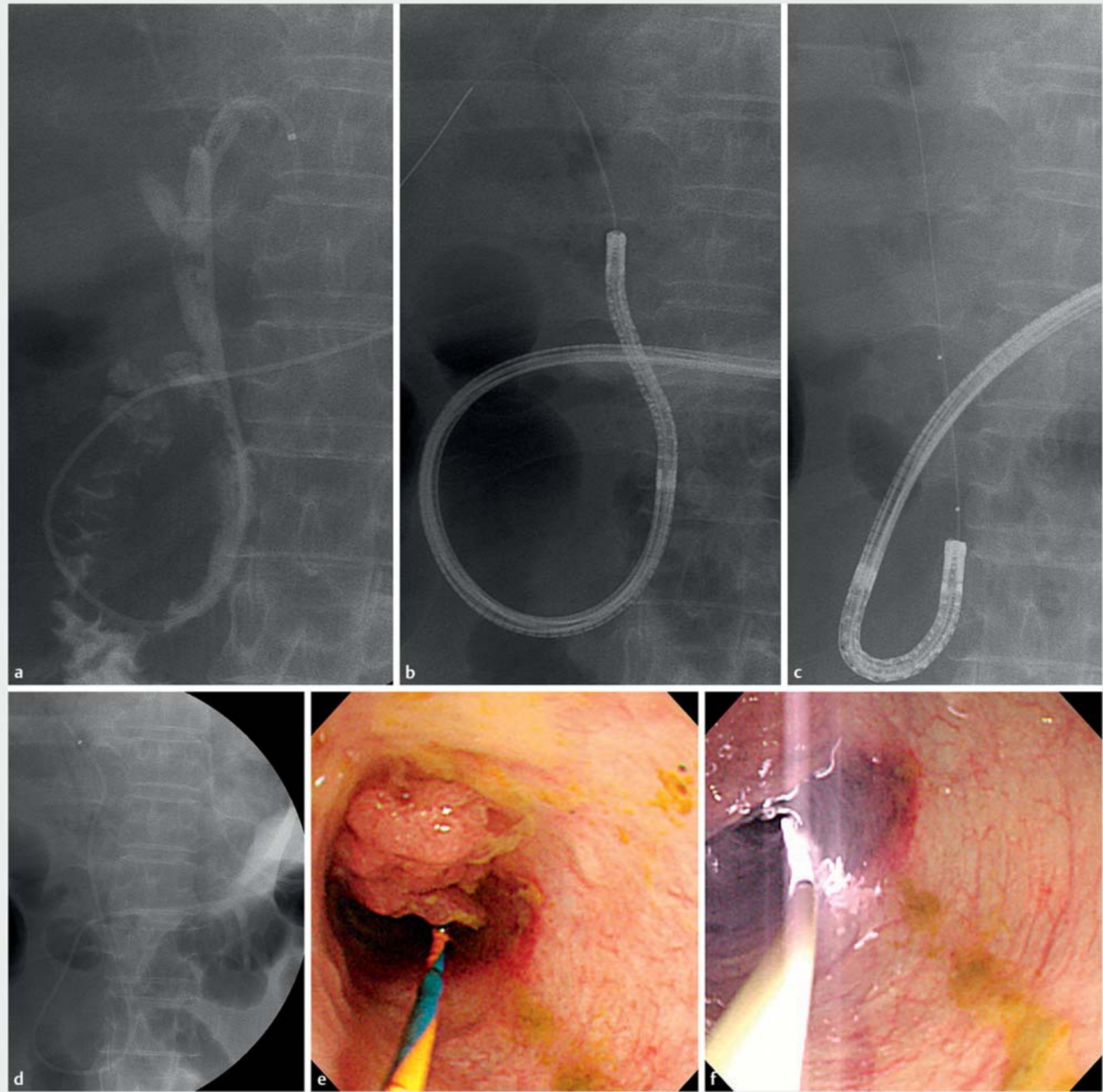

- Fig. 2 a Cholangiogram of a luminal-obstructing mass lesion. b Fluoroscopic view showing the advancement of an ultra-slim endoscope using intraductal balloon-guided method. c Fluoroscopic view showing the advancement of a photodynamic therapy (PDT) catheter using an ultra-slim endoscope into the bile duct. d A 5-Fr endoscopic nasobiliary drainage was inserted under direct peroral cholangioscopy (POC). e Cholangioscopic view showing bile duct cancer. f Direct POC-guided PDT.

Single-operator direct POC using an ultra-slim upper endoscope has been proposed. Direct POC allows high-quality narrow-band imaging (NBI) and has a larger working channel (up to $2.2 \mathrm{~mm}$ in diameter) for interventional procedures $[1,2]$. Thus, the limitations of cholangioscopy can be overcome by direct $\mathrm{POC}$ using a conventional ultra-slim upper endoscope. The 2.0-mm working channel of an ultra-slim endoscope enables direct placement of a $5 \mathrm{Fr}$ diameter plastic stent or drainage tube, intraductal lithotripsy, intraductal ablation therapy for bile duct tumors, and cholangioscopy-guided direct biliary drainage [1]. Direct POC also facilitates identification of the stricture site and direct drainage without changing the endoscope [7].

Our findings demonstrate the feasibility of selective biliary drainage under direct POC using an ultra-slim upper endoscope. The technical and functional success rates of biliary drainage were $100 \%$ in patients with a variety of clinical conditions, and minor bleeding after fragmentation of an intraductal stone by EHL was the only procedure-related AE. This indicates 

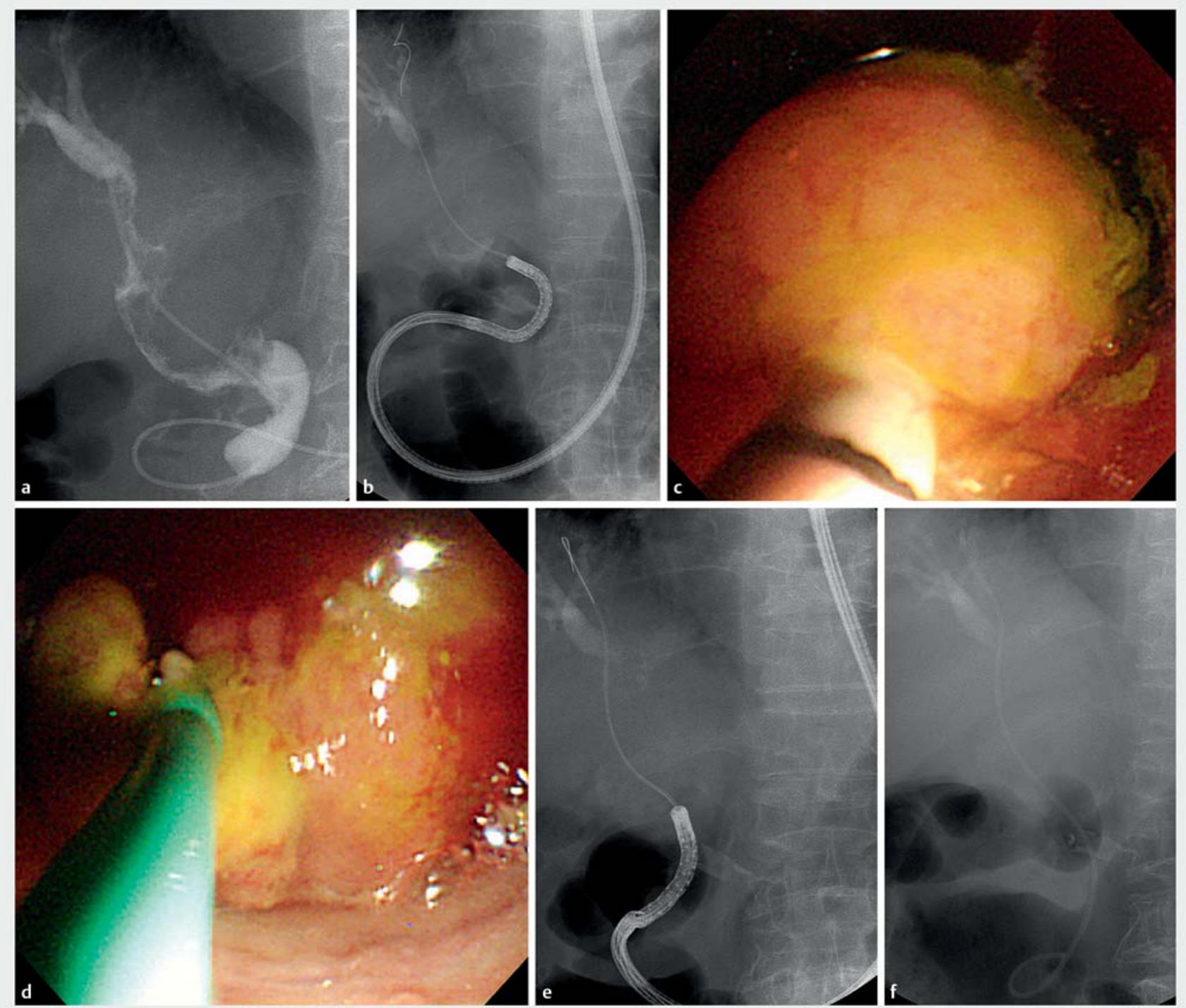

- Fig. 3 a Cholangiogram of indeterminate biliary stricture. b Fluoroscopic view showing the advancement of an ultra-slim endoscope into the bile duct and cannulation through the stricture. c Cholangioscopic view of intraductal mass. d A 5-Fr plastic stent was inserted under direct peroral cholangioscopy. e and $\mathbf{f}$ Fluoroscopic view showing selective cannulation and insertion of a 5 - Fr plastic stent.

the safety of selective biliary drainage under direct POC using an ultra-slim upper endoscope, which also requires only minimal insufflation with $\mathrm{CO} 2$ and administration of prophylactic antibiotics.

However, direct POC also has several limitations. First, direct $\mathrm{POC}$ can be performed only in patients with a dilated $C B D$ (>8 mm). Major sphincterotomy and/or papillary balloon dilation are needed for smooth insertion of the ultra-slim endoscope into the biliary tree. Second, only $5 \mathrm{Fr}$ plastic stents can be used due to the $2.0-\mathrm{mm}$ working channel of the ultra-slim endoscope; therefore, > 7-Fr conventional plastic stents cannot be used. In this study, direct POC was performed for the purpose of directly visualizing the extent of the lesion, biopsy, or PDT, especially in a malignant biliary stricture. Selective biliary drainage in this study was for biliary drainage to prevent devel- opment of cholangitis. Third, although intraductal balloonguided direct POC may be helpful, maintaining the position of the endoscope during the procedure is problematic. Fourth, cholangitis, pancreatitis can be additional procedure-related complications of direct POC. The most important procedurerelated complication of direct $\mathrm{POC}$ is possible of air embolism [12].

This study had several limitations. First, the retrospective cohort may have introduced selection bias. Second, the number of patients was relatively small and there was no control group, limiting the statistical power. Third, the two endoscopists who performed direct POC were experienced in both that procedure and endoscopic retrograde cholangiography. Thus, prospective studies involving operators with a standard- 
ized level of skill in direct POC are required to validate our findings.

\section{Conclusions}

In conclusion, selective biliary drainage under direct POC using an ultra-slim upper endoscope is feasible and safe for patients with a variety of clinical conditions. Although we have shown that drainage is feasible, it can only be used as a temporary measure to prevent the risk of cholangitis after direct POC. This is because only 5-Fr double-pigtail or straight plastic stent, and/or 5-Fr nasobiliary drainage catheters can be inserted through the POC system. Continuing development of the endoscopes and accessories is expected to facilitate the therapeutic application of direct POC.

Funding

This work was partly supported by SoonChunHyang University Research Fund.

Competing interests

The authors declare that they have no conflict of interest.

\section{References}

[1] Moon JH, Terheggen G, Choi HJ et al. Peroral cholangioscopy: diagnostic and therapeutic applications. Gastroenterology 2013; 144: $276-282$
[2] Lee $\mathrm{YN}$, Moon JH, Choi H] et al. A newly modified access balloon catheter for direct peroral cholangioscopy by using an ultraslim upper endoscope (with videos). Gastrointest Endosc 2016; 83: 240-247

[3] Moon JH, Ko BM, Choi HJ et al. Intraductal balloon-guided direct peroral cholangioscopy with an ultraslim upper endoscope (with videos). Gastrointest Endosc 2009; 70: 297-302

[4] Chandrasekhara V, Khashab MA, Muthusamy VR et al. Adverse events associated with ERCP. Gastrointest Endosc 2017; 85: 32-47

[5] Weinberg BM, Shindy W, Lo S. Endoscopic balloon sphincter dilation (sphincteroplasty) versus sphincterotomy for common bile duct stones. Cochrane Database Syst Rev 2006: Cd004890

[6] Freeman ML, Nelson DB, Sherman S et al. Complications of endoscopic biliary sphincterotomy. N Engl ] Med 1996; 335: 909-919

[7] Waxman I, Chennat J, Konda V. Peroral direct cholangioscopic-guided selective intrahepatic duct stent placement with an ultraslim endoscope. Gastrointest Endosc 2010; 71: 875-878

[8] Wright H, Sharma S, Gurakar A et al. Management of biliary stricture guided by the Spyglass Direct Visualization System in a liver transplant recipient: an innovative approach. Gastrointest Endosc 2008; 67: 1201-1203

[9] Kumar S. Cholangioscopy-directed endoscopic intervention for postliver transplantation anastomotic biliary stricture. Gastrointest Endosc 2015; 81: 1014-1015

[10] Woo YS, Lee JK, Noh DH et al. SpyGlass cholangioscopy-assisted guidewire placement for post-LDLT biliary strictures: a case series. Surgical Endoscopy 2016; 30: 3897-3903

[11] Shin JU, Lee JK, Kim KM et al. Endoscopic naso-gallbladder drainage by using cholangioscopy for acute cholecystitis combined with cholangitis or choledocholithiasis (with video). Gastrointest Endosc 2012; 76: 1052-1055

[12] Efthymiou M, Raftopoulos S, Antonio Chirinos J et al. Air embolism complicated by left hemiparesis after direct cholangioscopy with an intraductal balloon anchoring system. Gastrointest Endosc 2012; 75: $221-223$ 